\title{
Equivalent conditions and applications of a class of Hilbert-type integral inequalities involving multiple functions with quasi-homogeneous kernels
}

Junfei $\mathrm{CaO}^{1}$, Bing He${ }^{1}$, Yong Hong ${ }^{2^{*}}$ (D) and Bicheng Yang ${ }^{1}$

"Correspondence:
yonghonglbz@163.com
2College of Mathematics and
Statistics, Guangdong University of
Finance and Economics,
Guangzhou, P.R. China
Full list of author information is
available at the end of the article

available at the end of the article

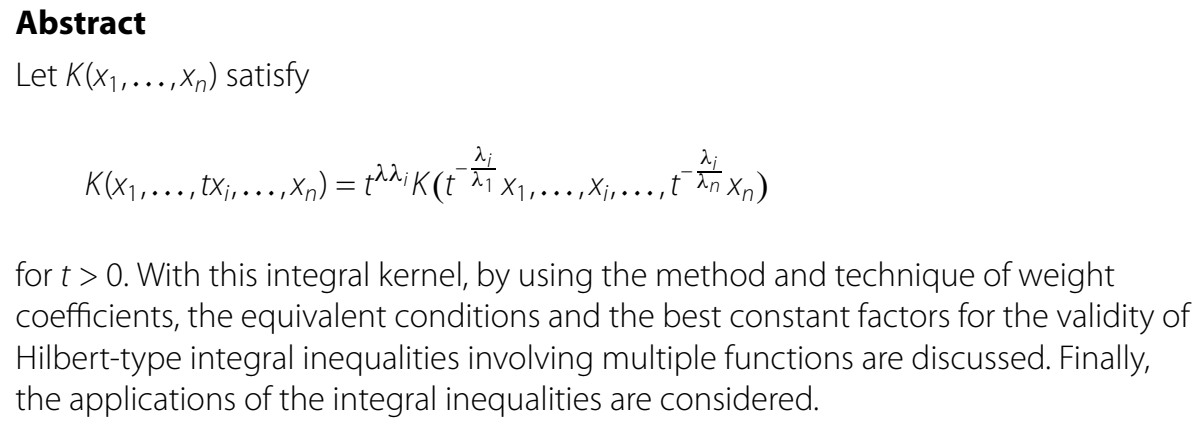

Let $K\left(x_{1}, \ldots, x_{n}\right)$ satisfy$$
K\left(x_{1}, \ldots, t x_{i}, \ldots, x_{n}\right)=t^{\lambda \lambda_{i}} K\left(t^{-\frac{\lambda_{i}}{\lambda_{1}}} x_{1}, \ldots, x_{i}, \ldots, t^{-\frac{\lambda_{i}}{\lambda_{n}}} x_{n}\right)
$$

for $t>0$. With this integral kernel, by using the method and technique of weight coefficients, the equivalent conditions and the best constant factors for the validity of Hilbert-type integral inequalities involving multiple functions are discussed. Finally, the applications of the integral inequalities are considered.

MSC: 26D15; 47A07

Keywords: Hilbert-type integral inequality; Quasi-homogeneous kernel; Equivalent conditions; Best constant factor

\section{Introduction}

Let $x=\left(x_{1}, \ldots, x_{n}\right), \mathbf{R}_{+}^{n}=\left\{x=\left(x_{1}, \ldots, x_{n}\right): x_{i}>0(i=1, \ldots, n)\right\}, r>1, f(t) \geq 0$, and $\alpha$ be a constant. Set

$$
L_{\alpha}^{r}(0,+\infty)=\left\{f(t):\|f\|_{r, \alpha}=\left(\int_{0}^{+\infty} t^{\alpha} f^{r}(t) d t\right)^{1 / r}<+\infty\right\}
$$

If $\sum_{i=1}^{n} \frac{1}{p_{i}}=1\left(p_{i}>1, i=1, \ldots, n\right), \alpha_{i} \in \mathbf{R}, f_{i}\left(x_{i}\right) \in L_{\alpha_{i}}^{p_{i}}(0,+\infty)(i=1, \ldots, n), K\left(x_{1}, \ldots, x_{n}\right) \geq 0$, $M$ is a constant, then we name the following inequality a Hilbert-type integral inequality:

$$
\int_{\mathbf{R}_{+}^{n}} K\left(x_{1}, \ldots, x_{n}\right) \prod_{i=1}^{n} f_{i}\left(x_{i}\right) d x_{1} \cdots d x_{n} \leq M \prod_{i=1}^{n}\left\|f_{i}\right\|_{p_{i}, \alpha_{i}} .
$$

An integral kernel $K\left(x_{1}, \ldots, x_{n}\right)$ is said to be a quasi-homogeneous function with parameters $\left(\lambda, \lambda_{1}, \ldots, \lambda_{n}\right)$ if, for $t>0$,

$$
K\left(x_{1}, \ldots, t x_{i}, \ldots, x_{n}\right)=t^{\lambda \lambda_{i}} K\left(t^{-\frac{\lambda_{i}}{\lambda_{1}}} x_{1}, \ldots, x_{i}, \ldots, t^{-\frac{\lambda_{i}}{\lambda_{n}}} x_{n}\right) \quad(i=1, \ldots, n) .
$$

\section{Springer}

(c) The Author(s) 2018. This article is distributed under the terms of the Creative Commons Attribution 4.0 International License (http://creativecommons.org/licenses/by/4.0/), which permits unrestricted use, distribution, and reproduction in any medium, provided you give appropriate credit to the original author(s) and the source, provide a link to the Creative Commons license, and indicate if changes were made. 
Obviously, $K\left(x_{1}, \ldots, x_{n}\right)$ becomes a homogeneous function of order $\lambda \lambda_{0}$ when $\lambda_{1}=\lambda_{2}=$ $\cdots=\lambda_{n}=\lambda_{0}$.

So far, many good results have been obtained in the study of Hilbert-type inequalities (cf. [1-24]). What are the necessary and sufficient conditions for the validity of a Hilberttype inequality? What is the best constant factor when the inequality holds? The research on such problems is undoubtedly of great significance to the study and applications of Hilbert-type inequality theory, but unfortunately, the research on this type of problems is rarely seen.

In this paper, we focus on the quasi-homogeneous integral kernels, discuss the equivalent conditions for the validity of Hilbert-type integral inequalities involving multiple functions, and obtain the expressions of the best constant factors when the inequalities are established. Finally, we discuss their applications.

\section{Some lemmas}

Lemma 1 Let integer $n \geq 2, \sum_{i=1}^{n} \frac{1}{p_{i}}=1\left(p_{i}>1, i=1, \ldots, n\right), \lambda \in \mathbf{R}, \alpha_{i} \in \mathbf{R}, \lambda_{i}>0$ (or $\lambda_{i}<$ $0)(i=1, \ldots, n)$, and $K\left(x_{1}, \ldots, x_{n}\right)$ be a nonnegative measurable function with parameters $\left(\lambda, \lambda_{1}, \ldots, \lambda_{n}\right), \sum_{i=1}^{n} \frac{\alpha_{i}+1}{\lambda_{i} p_{i}}=\lambda+\sum_{i=1}^{n} \frac{1}{\lambda_{i}}$. Set

$$
\begin{aligned}
W_{j}= & \int_{\mathbf{R}_{+}^{n-1}} K\left(u_{1}, \ldots, u_{j-1}, 1, u_{j+1}, \ldots, u_{n}\right) \\
& \times \prod_{i=1(i \neq j)}^{n} u_{i}^{-\frac{\alpha_{i}+1}{p_{i}}} d u_{1} \cdots d u_{j-1} d u_{j+1} \cdots d u_{n} .
\end{aligned}
$$

Then $\frac{1}{\lambda_{1}} W_{1}=\frac{1}{\lambda_{2}} W_{2}=\cdots=\frac{1}{\lambda n} W_{n}$, and

$$
\begin{aligned}
\omega_{j}\left(x_{j}\right) & =\int_{\mathbf{R}_{+}^{n-1}} K\left(x_{1}, \ldots, x_{n}\right) \prod_{i=1(i \neq j)}^{n} x_{i}^{-\frac{\alpha_{i}+1}{p_{i}}} d x_{1} \cdots d x_{j-1} d x_{j+1} \cdots d x_{n} \\
& =x_{j}^{\lambda_{j}\left(\lambda+\sum_{i=1(i \neq j)}^{n} \frac{\alpha_{i}+1}{\lambda_{i} p_{i}}+\sum_{i=1(i \neq j)}^{n} \frac{1}{\lambda_{i}}\right)} W_{j},
\end{aligned}
$$

where $j=1, \ldots, n$.

Proof When $j \geq 2$, by $\sum_{i=1}^{n} \frac{\alpha_{i}+1}{\lambda_{i} p_{i}}=\lambda+\sum_{i=1}^{n} \frac{1}{\lambda_{i}}$,

$$
\begin{aligned}
W_{j}= & \int_{\mathbf{R}_{+}^{n-1}} u_{1}^{\lambda \lambda_{1}} K\left(1, \frac{u_{2}}{u_{1}^{\frac{\lambda_{1}}{\lambda_{2}}}}, \ldots, \frac{u_{j-1}}{u_{1}^{\frac{\lambda_{1}}{\lambda_{j-1}}}}, \frac{1}{u_{1}^{\frac{\lambda_{1}}{\lambda_{j}}}}, \frac{u_{j+1}}{u_{1}^{\frac{\lambda_{1}}{\lambda_{j+1}}}}, \ldots, \frac{u_{n}}{u_{1}^{\frac{\lambda_{1}}{\lambda_{n}}}}\right) \\
& \times \prod_{i=1(i \neq j)}^{n} u_{i}^{-\frac{\alpha_{i}+1}{p_{i}}} d u_{1} \cdots d u_{j-1} d u_{j+1} \cdots d u_{n} \\
= & \frac{\lambda_{j}}{\lambda_{1}} \int_{\mathbf{R}_{+}^{n-1}} K\left(1, t_{2}, \ldots, t_{n}\right) t_{j}^{\lambda_{j}\left(-\lambda-\sum_{i=1}^{n} \frac{1}{\lambda_{i}}+\sum_{i=1(i \neq j)}^{n} \frac{\alpha_{i}+1}{\lambda_{i} p_{i}}\right)} \\
& \times \prod_{i=2(i \neq j)} t_{i}^{-\frac{\alpha_{i}+1}{p_{i}}} d t_{2} \cdots d t_{n} \\
= & \frac{\lambda_{j}}{\lambda_{1}} \int_{\mathbf{R}_{+}^{n-1}} K\left(1, t_{2}, \ldots, t_{n}\right) t_{j}^{\lambda_{j}\left(-\frac{\alpha_{j}+1}{\lambda_{j} p_{j}}\right)} \prod_{i=2(i \neq j)}^{n} t_{i}^{-\frac{\alpha_{i}+1}{p_{i}}} d t_{2} \cdots d t_{n}
\end{aligned}
$$




$$
=\frac{\lambda_{j}}{\lambda_{1}} \int_{\mathbf{R}_{+}^{n-1}} K\left(1, t_{2}, \ldots, t_{n}\right) \prod_{i=2}^{n} t_{i}^{-\frac{\alpha_{i}+1}{p_{i}}} d t_{2} \cdots d t_{n}=\frac{\lambda_{j}}{\lambda_{1}} W_{1} .
$$

Therefore $\frac{1}{\lambda_{j}} W_{j}=\frac{1}{\lambda_{1}} W_{1}(j \geq 2)$. When $j=1, \ldots, n$, we also get

$$
\begin{aligned}
& \omega_{j}\left(x_{j}\right)=\int_{\mathbf{R}_{+}^{n-1}} x_{j}^{\lambda \lambda_{j}} K\left(\frac{x_{1}}{x_{j}^{\frac{\lambda_{j}}{\lambda 1}}}, \ldots, \frac{x_{j-1}}{x_{j}^{\frac{\lambda_{j}}{\lambda_{j-1}}}}, 1, \frac{x_{j+1}}{x_{j}^{\frac{\lambda_{j}}{\lambda_{j+1}}}}, \ldots, \frac{x_{n}}{x_{j}^{\frac{\lambda_{j}}{\lambda n}}}\right) \\
& \times \prod_{i=1(i \neq j)}^{n} x_{i}^{-\frac{\alpha_{i}+1}{p_{i}}} d x_{1} \cdots d x_{j-1} d x_{j+1} \cdots d x_{n} \\
& =x_{j}^{\lambda \lambda_{j}-\lambda_{j} \sum_{i=1(i \neq j)}^{n} \frac{\alpha_{i}+1}{\lambda_{i} p_{i}}+\lambda_{j} \sum_{i=1(i \neq j)}^{n} \frac{1}{\lambda_{i}}} \\
& \times \int_{\mathbf{R}_{+}^{n-1}} K\left(u_{1}, \ldots, u_{j-1}, 1, u_{j+1}, \ldots, u_{n}\right) \\
& \times \prod_{i=1(i \neq j)}^{n} u_{i}^{-\frac{\alpha_{i}+1}{p_{i}}} d u_{1} \cdots d u_{j-1} d u_{j+1} \cdots d u_{n} \\
& =x_{j}^{\lambda_{j}\left(\lambda+\sum_{i=1(i \neq j)}^{n} \frac{\alpha_{i}+1}{\lambda_{i} p_{i}}+\sum_{i=1(i \neq j)}^{n} \frac{1}{\lambda_{i}}\right)} W_{j} .
\end{aligned}
$$

Lemma 2 ([25]) Let $p_{i}>0, a_{i}>0, \alpha_{i}>0(i=1, \ldots, n), \psi(u)$ be measurable. Then

$$
\begin{aligned}
\int & \cdots \int_{x_{i}>0,\left(\frac{x_{1}}{a_{1}}\right)^{\alpha_{1}}+\cdots+\left(\frac{x_{n}}{a_{n}}\right)^{\alpha_{n}} \leq 1} \psi\left(\left(\frac{x_{1}}{a_{1}}\right)^{\alpha_{1}}+\cdots+\left(\frac{x_{n}}{a_{n}}\right)^{\alpha_{n}}\right) \\
& \times x_{1}^{p_{1}-1} \cdots x_{n}^{p_{n}-1} d x_{1} \cdots d x_{n} \\
= & \frac{a_{1}^{p_{1}} \cdots a_{n}^{p_{n}} \Gamma\left(\frac{p_{1}}{\alpha_{1}}\right) \cdots \Gamma\left(\frac{p_{n}}{\alpha n}\right)}{\alpha_{1} \cdots \alpha_{n} \Gamma\left(\frac{p_{1}}{\alpha_{1}}+\cdots+\frac{p_{n}}{\alpha n}\right)} \int_{0}^{1} \psi(t) t^{\frac{p_{1}}{\alpha_{1}}+\cdots+\frac{p_{n}}{\alpha n}-1} d t,
\end{aligned}
$$

where $\Gamma$ represents the gamma function.

\section{Main results and their proofs}

Theorem 1 Suppose that $n \geq 2, \sum_{i=1}^{n} \frac{1}{p_{i}}=1\left(p_{i}>1\right), \lambda \in \mathbf{R}, \lambda_{i}>0$ (or $\left.\lambda_{i}<0\right), \alpha_{i} \in \mathbf{R}$ $(i=1, \ldots, n)$. If $K\left(x_{1}, \ldots, x_{n}\right)$ is a quasi-homogeneous positive function with parameters $\left(\lambda, \lambda_{1}, \ldots, \lambda_{n}\right)$, and

$$
W_{1}=\int_{\mathbf{R}_{+}^{n-1}} K\left(1, u_{2}, \ldots, u_{n}\right) \prod_{i=2}^{n} u_{i}^{-\frac{\alpha_{i}+1}{p_{i}}} d u_{2} \cdots d u_{n}
$$

is convergent, then

(i) the inequality

$$
\int_{\mathbf{R}_{+}^{n}} K\left(x_{1}, \ldots, x_{n}\right) \prod_{i=1}^{n} f_{i}\left(x_{i}\right) d x_{1} \cdots d x_{n} \leq M \prod_{i=1}^{n}\left\|f_{i}\right\|_{p_{i}, \alpha_{i}}
$$

holds for some constant $M>0$ if and only if $\sum_{i=1}^{n} \frac{\alpha_{i}+1}{\lambda_{i} p_{i}}=\lambda+\sum_{i=1}^{n} \frac{1}{\lambda_{i}}$, where $f_{i}\left(x_{i}\right) \in L_{\alpha_{i}}^{p_{i}}(0,+\infty)(i=1, \ldots, n)$; 
(ii) if (1) holds, then its best constant factor is $\inf M=\frac{W_{1}}{\left|\lambda_{1}\right|} \prod_{i=1}^{n}\left|\lambda_{i}\right|^{1 / p_{i}}$.

Proof (i) Sufficiency. Assume that $\sum_{i=1}^{n} \frac{\alpha_{i}+1}{\lambda_{i} p_{i}}=\lambda+\sum_{i=1}^{n} \frac{1}{\lambda_{i}}$. Since

$$
\prod_{j=1}^{n} x_{j}^{\frac{\alpha_{j}+1}{p_{j}}}\left(\prod_{i=1}^{n} x_{i}^{-\frac{\alpha_{i}+1}{p_{i}}}\right)^{1 / p_{j}}=\prod_{j=1}^{n} x_{j}^{\frac{\alpha_{j}+1}{p_{j}}} \prod_{i=1}^{n} x_{i}^{-\frac{\alpha_{i}+1}{p_{i}} \sum_{k=1}^{n} \frac{1}{p_{k}}}=1
$$

by Hölder's inequality and Lemma 1, we obtain

$$
\begin{aligned}
& \int_{\mathbf{R}_{+}^{n}} K\left(x_{1}, \ldots, x_{n}\right) \prod_{i=1}^{n} f_{i}\left(x_{i}\right) d x_{1} \cdots d x_{n} \\
& =\int_{\mathbf{R}_{+}^{n}} K\left(x_{1}, \ldots, x_{n}\right)\left[\prod_{j=1}^{n} x_{j}^{\frac{\alpha_{j}+1}{p_{j}}}\left(\prod_{i=1}^{n} x_{i}^{-\frac{\alpha_{i}+1}{p_{i}}}\right)^{1 / p_{j}} f_{j}\left(x_{j}\right)\right] d x_{1} \cdots d x_{n} \\
& \leq \prod_{j=1}^{n}\left[\int_{\mathbf{R}_{+}^{n}} x_{j}^{\alpha_{j}+1}\left(\prod_{i=1}^{n} x_{i}^{-\frac{\alpha_{i}+1}{p_{i}}}\right) f_{j}^{p_{j}}\left(x_{j}\right) K\left(x_{1}, \ldots, x_{n}\right) d x_{1} \cdots d x_{n}\right]^{1 / p_{j}} \\
& =\prod_{j=1}^{n}\left[\int_{0}^{+\infty} x_{j}^{\alpha_{j}+1-\frac{\alpha_{j}+1}{p_{j}}} f_{j}^{p_{j}}\left(x_{j}\right)\right. \\
& \left.\times\left(\int_{\mathbf{R}_{+}^{n-1}} K\left(x_{1}, \ldots, x_{n}\right) \prod_{i=1(i \neq j)}^{n} x_{i}^{-\frac{\alpha_{i}+1}{p_{i}}} d x_{1} \cdots d x_{j-1} d x_{j+1} \cdots d x_{n}\right) d x_{j}\right]^{1 / p_{j}} \\
& =\prod_{j=1}^{n}\left(\int_{0}^{+\infty} x_{j}^{\alpha_{j}+1-\frac{\alpha_{j}+1}{p_{j}}} f_{j}^{p_{j}}\left(x_{j}\right) \omega_{j}\left(x_{j}\right) d x_{j}\right)^{1 / p_{j}} \\
& =\prod_{j=1}^{n}\left[\int_{0}^{+\infty} x_{j}^{\alpha_{j}+1-\frac{\alpha_{j}+1}{p_{j}}+\lambda_{j}\left(\lambda-\sum_{i=1(i \neq j)}^{n} \frac{\alpha_{i}+1}{\lambda_{i} p_{i}}+\sum_{i=1(i \neq j)}^{n} \frac{1}{\lambda_{i}}\right)} f_{j}^{p_{j}}\left(x_{j}\right) W_{j} d x_{j}\right]^{1 / p_{j}} \\
& =\prod_{j=1}^{n} W_{j}^{1 / p_{j}} \prod_{j=1}^{n}\left[\int_{0}^{+\infty} x_{j}^{\lambda_{j}\left(\frac{\alpha_{j}}{\lambda_{j}}-\sum_{i=1}^{n} \frac{\alpha_{i}+1}{\lambda_{i} p_{i}}+\lambda+\sum_{i=1}^{n} \frac{1}{\lambda_{i}}\right)} f_{j}^{p_{j}}\left(x_{j}\right) d x_{j}\right]^{1 / p_{j}} \\
& =\prod_{i=1}^{n} W_{i}^{1 / p_{i}} \prod_{i=1}^{n}\left(\int_{0}^{+\infty} x_{i}^{\alpha_{i}} f_{i}^{p_{i}}\left(x_{i}\right) d x_{i}\right)^{1 / p_{i}} \\
& =\frac{W_{1}}{\left|\lambda_{1}\right|}\left(\prod_{i=1}^{n}\left|\lambda_{i}\right|^{1 / p_{i}}\right) \prod_{i=1}^{n}\left\|f_{i}\right\|_{p_{i}, \alpha_{i}},
\end{aligned}
$$

thus (1) holds when taking any constant $M \geq \frac{W_{1}}{\left|\lambda_{1}\right|} \prod_{i=1}^{n}\left|\lambda_{i}\right|^{1 / p_{i}}$.

Necessity. Assume that (1) holds. Set $c=\sum_{i=1}^{n} \frac{\alpha_{i}+1}{\lambda_{i} p_{i}}-\lambda-\sum_{i=1}^{n} \frac{1}{\lambda_{i}}$. Next we will prove $c=0$.

First consider the case of $\lambda_{i}>0(i=1, \ldots, n)$. If $c>0$, for $0<\varepsilon<c$, take

$$
f_{i}\left(x_{i}\right)= \begin{cases}x_{i}^{\left(-\alpha_{i}-1+\lambda_{i} \varepsilon\right) / p_{i}}, & 0<x_{i} \leq 1 \\ 0, & x_{i}>1\end{cases}
$$


where $i=1, \ldots, n$. Then

$$
\begin{aligned}
& \prod_{i=1}^{n}\left\|f_{i}\right\|_{p_{i}, \alpha_{i}}=\prod_{i=1}^{n}\left(\int_{0}^{1} x_{i}^{-1+\lambda_{i} \varepsilon} d x_{i}\right)^{1 / p_{i}}=\frac{1}{\varepsilon} \prod_{i=1}^{n}\left(\frac{1}{\lambda_{i}}\right)^{1 / p_{i}}, \\
& \int_{\mathbf{R}_{+}^{n}} K\left(x_{1}, \ldots, x_{n}\right) \prod_{i=1}^{n} f_{i}\left(x_{i}\right) d x_{1} \cdots d x_{n} \\
& =\int_{0}^{1} x_{1}^{\left(-\alpha_{1}-1+\lambda_{1} \varepsilon\right) / p_{1}} \\
& \times\left(\int_{0}^{1} \cdots \int_{0}^{1} K\left(x_{1}, x_{2}, \ldots, x_{n}\right) \prod_{i=2}^{n} x_{i}^{\left(-\alpha_{i}-1+\lambda_{i} \varepsilon\right) / p_{i}} d x_{2} \cdots d x_{n}\right) d x_{1} \\
& =\int_{0}^{1} x_{1}^{\left(-\alpha_{1}-1+\lambda_{1} \varepsilon\right) / p_{1}+\lambda \lambda_{1}}\left(\int_{0}^{1} \cdots \int_{0}^{1} K\left(1, x_{1}^{-\frac{\lambda_{1}}{\lambda_{2}}} x_{2}, \ldots, x_{1}^{-\frac{\lambda_{1}}{\lambda n}} x_{n}\right)\right. \\
& \left.\times \prod_{i=2}^{n} x_{i}^{\left(-\alpha_{i}-1+\lambda_{i} \varepsilon\right) / p_{i}} d x_{2} \cdots d x_{n}\right) d x_{1} \\
& =\int_{0}^{1} x_{1}^{-\frac{\alpha_{1}+1-\lambda_{1} \varepsilon}{p_{1}}+\lambda \lambda_{1}+\lambda_{1} \sum_{i=2}^{n} \frac{1}{\lambda_{i}}-\lambda_{1} \sum_{i=2}^{n} \frac{\alpha_{i}+1-\lambda_{i} \varepsilon}{\lambda_{i} p_{i}}}\left(\int_{0}^{x_{1}^{-\lambda_{1} / \lambda_{2}}} \cdots \int_{0}^{x_{n}^{-\lambda_{1} / \lambda_{n}}}\right. \\
& \left.\times K\left(1, u_{2}, \ldots, u_{n}\right) \prod_{i=2}^{n} u_{i}^{\left(-\alpha_{i}-1+\lambda_{i} \varepsilon\right) / p_{i}} d u_{2} \cdots d u_{n}\right) d x_{1} \\
& =\int_{0}^{1} x_{1}^{\lambda_{1}\left(\lambda+\sum_{i=1}^{n} \frac{1}{\lambda_{i}}-\sum_{i=1}^{n} \frac{\alpha_{i}+1}{\lambda_{i} p_{i}}-\frac{1}{\lambda_{1}}+\varepsilon\right)}\left(\int_{0}^{x_{1}^{-\lambda_{1} / \lambda_{2}}} \cdots \int_{0}^{x_{n}^{-\lambda_{1} / \lambda_{n}}}\right. \\
& \left.\times K\left(1, u_{2}, \ldots, u_{n}\right) \prod_{i=2}^{n} u_{i}^{\left(-\alpha_{i}-1+\lambda_{i} \varepsilon\right) / p_{i}} d u_{2} \cdots d u_{n}\right) d x_{1} \\
& \geq \int_{0}^{1} x_{1}^{-1-\lambda_{1} c+\lambda_{1} \varepsilon} d x_{1}\left(\int_{0}^{1} \ldots \int_{0}^{1} K\left(1, u_{2}, \ldots, u_{n}\right)\right. \\
& \left.\times \prod_{i=2}^{n} u_{i}^{\left(-\alpha_{i}-1+\lambda_{i} \varepsilon\right) / p_{i}} d u_{2} \cdots d u_{n}\right)
\end{aligned}
$$

It follows from (1), (2), and (3) that

$$
\begin{aligned}
& \int_{0}^{1} x_{1}^{-1-\lambda_{1} c+\lambda_{1} \varepsilon} d x_{1}\left(\int_{0}^{1} \cdots \int_{0}^{1} K\left(1, u_{2}, \ldots, u_{n}\right) \prod_{i=2}^{n} u_{i}^{\left(-\alpha_{i}-1+\lambda_{i} \varepsilon\right) / p_{i}} d u_{2} \cdots d u_{n}\right) \\
& \quad \leq \frac{M}{\varepsilon} \prod_{i=1}^{n}\left(\frac{1}{\lambda_{i}}\right)^{1 / p_{i}} .
\end{aligned}
$$

Since $-1-\lambda_{1} c+\lambda_{1} \varepsilon<-1, \int_{0}^{1} x_{1}^{-1-\lambda_{1} c+\lambda_{1} \varepsilon} d x_{1}$ diverges to $+\infty$. Whence it is a contradiction to (4). In other words, it is not valid for $c>0$. 
If $c<0$, for $0<\varepsilon<-c$, take

$$
f_{i}\left(x_{i}\right)= \begin{cases}x_{i}^{\left(-\alpha_{i}-1-\lambda_{i} \varepsilon\right) / p_{i}}, & x_{i} \geq 1 \\ 0, & 0<x_{i}<1\end{cases}
$$

where $i=1, \ldots, n$. Similarly, we get

$$
\begin{aligned}
& \int_{1}^{+\infty} x_{1}^{-1+\lambda_{1} c-\lambda_{1} \varepsilon} d x_{1} \\
& \quad \times\left(\int_{1}^{+\infty} \cdots \int_{1}^{+\infty} K\left(1, u_{2}, \ldots, u_{n}\right) \prod_{i=2}^{n} u_{i}^{\left(-\alpha_{i}-1-\lambda_{i} \varepsilon\right) / p_{i}} d u_{2} \cdots d u_{n}\right) \\
& \quad \leq \frac{M}{\varepsilon} \prod_{i=1}^{n}\left(\frac{1}{\lambda_{i}}\right)^{1 / p_{i}} .
\end{aligned}
$$

Since $-1-\lambda_{1} c+\lambda_{1} \varepsilon>-1$ and $\int_{1}^{+\infty} x_{1}^{-1-\lambda_{1} c-\lambda_{1} \varepsilon} d x_{1}$ diverges to $+\infty$, which contradicts the above inequality, hence it does not hold for $c<0$.

To sum up, we have $c=0$ for $\lambda_{i}>0(i=1, \ldots, n)$.

Now let us consider the case of $\lambda_{i}<0(i=1, \ldots, n)$. If $c>0$, for $0<\varepsilon<c$, take

$$
f_{i}\left(x_{i}\right)= \begin{cases}x_{i}^{\left(-\alpha_{i}-1+\lambda_{i} \varepsilon\right) / p_{i}}, & x_{i} \geq 1 \\ 0, & 0<x_{i}<1\end{cases}
$$

where $i=1, \ldots, n$. Consequently,

$$
\begin{aligned}
& \prod_{i=1}^{n}\left\|f_{i}\right\|_{p_{i}, \alpha_{i}}=\prod_{i=1}^{n}\left(\int_{1}^{+\infty} x_{i}^{-1+\lambda_{i} \varepsilon} d x_{i}\right)^{1 / p_{i}}=\frac{1}{\varepsilon} \prod_{i=1}^{n}\left(\frac{1}{-\lambda_{i}}\right)^{1 / p_{i}}, \\
& \int_{\mathbf{R}_{+}^{n}} K\left(x_{1}, \ldots, x_{n}\right) \prod_{i=1}^{n} f_{i}\left(x_{i}\right) d x_{1} \cdots d x_{n} \\
& =\int_{1}^{+\infty} x_{1}^{\left(-\alpha_{1}-1+\lambda_{1} \varepsilon\right) / p_{1}}\left(\int_{1}^{+\infty} \cdots \int_{1}^{+\infty} x_{1}^{\lambda \lambda_{1}} K\left(1, x_{1}^{-\frac{\lambda_{1}}{\lambda_{2}}} x_{2}, \ldots, x_{1}^{-\frac{\lambda_{1}}{\lambda_{n}}} x_{n}\right)\right. \\
& \left.\times \prod_{i=2}^{n} x_{i}^{\left(-\alpha_{i}-1+\lambda_{i} \varepsilon\right) / p_{i}} d x_{2} \cdots d x_{n}\right) d x_{1} \\
& =\int_{1}^{+\infty} x_{1}^{-\frac{\alpha_{1}+1-\lambda_{1} \varepsilon}{p_{1}}+\lambda \lambda_{1}+\lambda_{1} \sum_{i=2}^{n} \frac{1}{\lambda_{i}}-\lambda_{1} \sum_{i=2}^{n} \frac{\alpha_{i}+1-\lambda_{i} \varepsilon}{\lambda_{i} p_{i}}}\left(\int_{x_{1}^{-\lambda_{1} / \lambda_{2}}}^{+\infty} \cdots \int_{x_{n}^{-\lambda_{1} / \lambda_{n}}}^{+\infty}\right. \\
& \left.\times K\left(1, u_{2}, \ldots, u_{n}\right) \prod_{i=2}^{n} u_{i}^{\left(-\alpha_{i}-1+\lambda_{i} \varepsilon\right) / p_{i}} d u_{2} \cdots d u_{n}\right) d x_{1} \\
& \geq \int_{1}^{+\infty} x_{1}^{-1-\lambda_{1} c+\lambda_{1} \varepsilon} d x_{1}\left(\int_{1}^{+\infty} \cdots \int_{1}^{+\infty} K\left(1, u_{2}, \ldots, u_{n}\right)\right. \\
& \left.\times \prod_{i=2}^{n} u_{i}^{\left(-\alpha_{i}-1+\lambda_{i} \varepsilon\right) / p_{i}} d u_{2} \cdots d u_{n}\right)
\end{aligned}
$$


It follows from (1), (6), and (7) that

$$
\begin{aligned}
& \int_{1}^{+\infty} x_{1}^{-1-\lambda_{1} c+\lambda_{1} \varepsilon} d x_{1} \\
& \quad \times\left(\int_{1}^{+\infty} \cdots \int_{1}^{+\infty} K\left(1, u_{2}, \ldots, u_{n}\right) \prod_{i=2}^{n} u_{i}^{\left(-\alpha_{i}-1+\lambda_{i} \varepsilon\right) / p_{i}} d u_{2} \cdots d u_{n}\right) \\
& \quad \leq \frac{M}{\varepsilon} \prod_{i=1}^{n}\left(\frac{1}{-\lambda_{i}}\right)^{1 / p_{i}} .
\end{aligned}
$$

Since $-1-\lambda_{1} c+\lambda_{1} \varepsilon>-1, \int_{1}^{+\infty} x_{1}^{-1-\lambda_{1} c+\lambda_{1} \varepsilon} d x_{1}$ diverges to $+\infty$. Thus it is a contradiction to the above inequality. That is, it does not hold for $c>0$.

$$
\text { If } c<0 \text {, for } 0<\varepsilon<-c \text {, take }
$$

$$
f_{i}\left(x_{i}\right)= \begin{cases}x_{i}^{\left(-\alpha_{i}-1-\lambda_{i} \varepsilon\right) / p_{i}}, & 0<x_{i} \leq 1 \\ 0, & x_{i}>1\end{cases}
$$

where $i=1, \ldots, n$. Similarly, one can get

$$
\begin{aligned}
& \int_{0}^{1} x_{1}^{-1-\lambda_{1} c-\lambda_{1} \varepsilon} d x_{1}\left(\int_{0}^{1} \cdots \int_{0}^{1} K\left(1, u_{2}, \ldots, u_{n}\right)\right. \\
& \left.\quad \times \prod_{i=2}^{n} u_{i}^{\left(-\alpha_{i}-1-\lambda_{i} \varepsilon\right) / p_{i}} d u_{2} \cdots d u_{n}\right) \\
& \leq \frac{M}{\varepsilon} \prod_{i=1}^{n}\left(\frac{1}{-\lambda_{i}}\right)^{1 / p_{i}} .
\end{aligned}
$$

Since $-1-\lambda_{1} c-\lambda_{1} \varepsilon<-1, \int_{0}^{1} x_{1}^{-1-\lambda_{1} c-\lambda_{1} \varepsilon} d x_{1}$ diverges to $+\infty$, which also contradicts the above inequality. It does not hold for $c<0$.

To sum up, we also get $c=0$ for $\lambda_{i}<0(i=1, \ldots, n)$.

(ii) Suppose that (1) holds. If the constant factor inf $M \neq \frac{W_{1}}{\left|\lambda_{1}\right|} \prod_{i=1}^{n}\left|\lambda_{i}\right|^{1 / p_{i}}$, then there exists a constant $M_{0}<\frac{W_{1}}{\left|\lambda_{1}\right|} \prod_{i=1}^{n}\left|\lambda_{i}\right|^{1 / p_{i}}$ such that

$$
\int_{\mathbf{R}_{+}^{n}} K\left(x_{1}, \ldots, x_{n}\right) \prod_{i=1}^{n} f_{i}\left(x_{i}\right) d x_{1} \cdots d x_{n} \leq M_{0} \prod_{i=1}^{n}\left\|f_{i}\right\|_{p_{i}, \alpha_{i}} .
$$

For sufficiently small $\varepsilon>0$ and $\delta>0$, take

$$
f_{1}\left(x_{1}\right)= \begin{cases}x_{1}^{\left(-\alpha_{1}-1-\left|\lambda_{1}\right| \varepsilon\right) / p_{1}}, & x_{1} \geq 1 \\ 0, & 0<x_{1}<1\end{cases}
$$

For $i=2,3, \ldots, n$, take

$$
f_{i}\left(x_{i}\right)= \begin{cases}x_{i}^{\left(-\alpha_{i}-1-\left|\lambda_{i}\right| \varepsilon\right) / p_{i}}, & x_{i} \geq \delta \\ 0, & 0<x_{i}<\delta\end{cases}
$$


Therefore,

$$
\begin{aligned}
& \prod_{i=1}^{n}\left\|f_{i}\right\|_{p_{i}, \alpha_{i}} \\
& =\left(\int_{1}^{+\infty} x_{1}^{-1-\left|\lambda_{1}\right| \varepsilon} d x_{1}\right)^{1 / p_{1}} \prod_{i=2}^{n}\left(\int_{\delta}^{+\infty} x_{i}^{1-\left|\lambda_{i}\right| \varepsilon} d x_{i}\right)^{1 / p_{i}} \\
& =\left(\frac{1}{\left|\lambda_{1}\right| \varepsilon}\right)^{1 / p_{1}} \prod_{i=2}^{n}\left(\frac{1}{\left|\lambda_{i}\right| \varepsilon} \cdot \frac{1}{\delta^{\left|\lambda_{i}\right| \varepsilon}}\right)^{1 / p_{i}} \\
& =\frac{1}{\varepsilon} \prod_{i=1}^{n}\left(\frac{1}{\left|\lambda_{i}\right|}\right)^{1 / p_{i}} \prod_{i=2}^{n}\left(\frac{1}{\delta^{\left|\lambda_{i}\right| \varepsilon}}\right)^{1 / p_{i}} \\
& \int_{\mathbf{R}_{+}^{n}} K\left(x_{1}, \ldots, x_{n}\right) \prod_{i=1}^{n} f_{i}\left(x_{i}\right) d x_{1} \cdots d x_{n} \\
& =\int_{0}^{1} x_{1}^{-\frac{\alpha_{1}+1+\left|\lambda_{1}\right| \varepsilon}{p_{1}}}\left(\int_{\delta}^{+\infty} \cdots \int_{\delta}^{+\infty} K\left(x_{1}, \ldots, x_{n}\right)\right. \\
& \left.\times \prod_{i=2}^{n} x_{i}^{-\frac{\alpha_{i}+1+\left|\lambda_{i}\right| \varepsilon}{p_{i}}} d x_{2} \cdots d x_{n}\right) d x_{1} \\
& =\int_{0}^{1} x_{1}^{\lambda \lambda_{1}-\frac{\alpha_{1}+1+\left|\lambda_{1}\right| \varepsilon}{p_{1}}}\left(\int_{\delta}^{+\infty} \cdots \int_{\delta}^{+\infty} K\left(1, x_{1}^{-\lambda_{1} / \lambda_{2}} x_{2}, \ldots, x_{1}^{-\lambda_{1} / \lambda_{n}} x_{n}\right)\right. \\
& \left.\times \prod_{i=2}^{n} x_{i}^{-\frac{\alpha_{i}+1+\left|\lambda_{i}\right| \varepsilon}{p_{i}}} d x_{2} \cdots d x_{n}\right) d x_{1}
\end{aligned}
$$

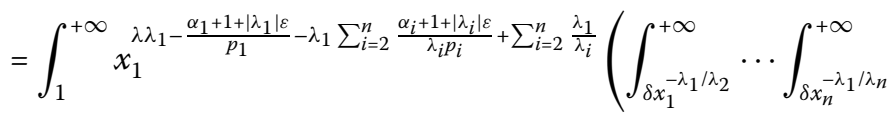

$$
\begin{aligned}
& \left.\times K\left(1, u_{2}, \ldots, u_{n}\right) \prod_{i=2}^{n} u_{i}^{-\frac{\alpha_{i}+1+\left|\lambda_{i}\right| \varepsilon}{p_{i}}} d u_{2} \cdots d u_{n}\right) d x_{1} \\
& \geq \int_{1}^{+\infty} x_{1}^{\lambda_{1}\left(\lambda-\sum_{i=1}^{n} \frac{\alpha_{i}+1}{\lambda_{i} p_{i}}+\sum_{i=1}^{n} \frac{1}{\lambda_{i}}-\frac{1}{\lambda_{1}}-\sum_{i=1}^{n} \frac{\left|\lambda_{i}\right| \varepsilon}{\lambda_{i} p_{i}}\right)} d x_{1}\left(\int_{\delta}^{+\infty} \cdots \int_{\delta}^{+\infty}\right. \\
& \left.\times K\left(1, u_{2}, \ldots, u_{n}\right) \prod_{i=2}^{n} u_{i}^{-\frac{\alpha_{i}+1+\left|\lambda_{i}\right| \varepsilon}{p_{i}}} d u_{2} \cdots d u_{n}\right) \\
& =\int_{1}^{+\infty} x_{1}^{-1-\left|\lambda_{1}\right| \varepsilon} d x_{1}\left(\int_{\delta}^{+\infty} \cdots \int_{\delta}^{+\infty} K\left(1, u_{2}, \ldots, u_{n}\right) \times \prod_{i=2}^{n} u_{i}^{-\frac{\alpha_{i}+1+\left|\lambda_{i}\right| \varepsilon}{p_{i}}} d u_{2} \cdots d u_{n}\right) \\
& =\frac{1}{\left|\lambda_{1}\right| \varepsilon} \int_{\delta}^{+\infty} \cdots \int_{\delta}^{+\infty} K\left(1, u_{2}, \ldots, u_{n}\right) \prod_{i=2}^{n} u_{i}^{-\frac{\alpha_{i}+1+\left|\lambda_{i}\right| \varepsilon}{p_{i}}} d u_{2} \cdots d u_{n} .
\end{aligned}
$$

It follows from (1), (10), and (11) that

$$
\frac{1}{\left|\lambda_{1}\right|} \int_{\delta}^{+\infty} \cdots \int_{\delta}^{+\infty} K\left(1, u_{2}, \ldots, u_{n}\right) \prod_{i=2}^{n} u_{i}^{-\frac{\alpha_{i}+1+\left|\lambda_{i}\right| \varepsilon}{p_{i}}} d u_{2} \cdots d u_{n}
$$




$$
\leq M_{0} \prod_{i=1}^{n}\left(\frac{1}{\left|\lambda_{i}\right|}\right)^{1 / p_{i}} \prod_{i=2}^{n}\left(\frac{1}{\delta^{\left|\lambda_{i}\right| \varepsilon}}\right)^{1 / p_{i}}
$$

Consequently,

$$
\frac{1}{\left|\lambda_{1}\right|} \prod_{i=1}^{n}\left|\lambda_{i}\right|^{1 / p_{i}} \int_{\delta}^{+\infty} \cdots \int_{\delta}^{+\infty} K\left(1, u_{2}, \ldots, u_{n}\right) \prod_{i=2}^{n} u_{i}^{-\left(\alpha_{i}+1\right) / p_{i}} d u_{2} \cdots d u_{n} \leq M_{0}
$$

as $\varepsilon \rightarrow 0^{+}$. And then let $\delta \rightarrow 0^{+}$, we eventually get

$$
\begin{aligned}
& \frac{W_{1}}{\left|\lambda_{1}\right|} \prod_{i=1}^{n}\left|\lambda_{i}\right|^{1 / p_{i}} \\
& \quad=\frac{1}{\left|\lambda_{1}\right|} \prod_{i=1}^{n}\left|\lambda_{i}\right|^{1 / p_{i}} \int_{\mathbf{R}_{+}^{n-1}} K\left(1, u_{2}, \ldots, u_{n}\right) \prod_{i=2}^{n} u_{i}^{-\left(\alpha_{i}+1\right) / p_{i}} d u_{2} \cdots d u_{n} \\
& \quad \leq M_{0}
\end{aligned}
$$

this is a contradiction. Hence $\inf M=\frac{W_{1}}{\left|\lambda_{1}\right|} \prod_{i=1}^{n}\left|\lambda_{i}\right|^{1 / p_{i}}$, i.e., the constant factor $\frac{W_{1}}{\left|\lambda_{1}\right|} \prod_{i=1}^{n}\left|\lambda_{i}\right|^{1 / p_{i}}$ is the best.

\section{Applications}

Theorem 2 Suppose that $n \geq 2, \sum_{i=1}^{n} \frac{1}{p_{i}}=1\left(p_{i}>1\right), \lambda_{i}>0\left(\right.$ or $\left.\lambda_{i}<0\right), f_{i}\left(x_{i}\right) \in L_{p_{i}-1}^{p_{i}}(0,+\infty)$, $i=1, \ldots, n$. Then

$$
\int_{\mathbf{R}_{+}^{n}} \frac{\min \left\{x_{1}^{\lambda_{1}}, \ldots, x_{n}^{\lambda_{n}}\right\}}{\max \left\{x_{1}^{\lambda_{1}}, \ldots, x_{n}^{\lambda_{n}}\right\}} \prod_{i=1}^{n} f_{i}\left(x_{i}\right) d x_{1} \cdots d x_{n} \leq\left(n ! \prod_{i=1}^{n}\left|\lambda_{i}\right|^{\frac{1}{p_{i}}-1}\right) \prod_{i=1}^{n}\left\|f_{i}\right\|_{p_{i}, p_{i}-1},
$$

where the constant factor is the best.

Proof Set $\alpha_{i}=p_{i}-1, \lambda=0$, then $\sum_{i=1}^{n} \frac{\alpha_{i}+1}{\lambda_{i} p_{i}}=\lambda+\sum_{i=1}^{n} \frac{1}{\lambda_{i}}$. Take

$$
K\left(x_{1}, \ldots, x_{n}\right)=\frac{\min \left\{x_{1}^{\lambda_{1}}, \ldots, x_{n}^{\lambda_{n}}\right\}}{\max \left\{x_{1}^{\lambda_{1}}, \ldots, x_{n}^{\lambda_{n}}\right\}}
$$

then $K\left(x_{1}, \ldots, x_{n}\right)$ is a quasi-homogeneous positive function with parameters $\left(\lambda, \lambda_{1}, \ldots, \lambda_{n}\right)$, and

$$
\begin{aligned}
W_{1} & =\int_{\mathbf{R}_{+}^{n-1}} K\left(1, u_{2}, \ldots, u_{n}\right) \prod_{i=2}^{n} u_{i}^{-\left(\alpha_{i}+1\right) / p_{i}} d u_{2} \cdots d u_{n} \\
& =\int_{\mathbf{R}_{+}^{n-1}} \frac{\min \left\{1, u_{2}^{\lambda_{2}}, \ldots, u_{n}^{\lambda_{n}}\right\}}{\max \left\{1, u_{2}^{\lambda_{2}}, \ldots, u_{n}^{\lambda_{n}}\right\}} \prod_{i=2}^{n} u_{i}^{-1} d u_{2} \cdots d u_{n} \\
& =\prod_{i=2}^{n} \frac{1}{\left|\lambda_{i}\right|} \int_{\mathbf{R}_{+}^{n-1}} \frac{\min \left\{1, t_{2}, \ldots, t_{n}\right\}}{\max \left\{1, t_{2}, \ldots, t_{n}\right\}} \prod_{i=2}^{n} t_{i}^{-1} d t_{2} \cdots d t_{n} .
\end{aligned}
$$

In view of [1], we get

$$
\int_{\mathbf{R}_{+}^{n-1}} \frac{\min \left\{1, t_{2}, \ldots, t_{n}\right\}}{\max \left\{1, t_{2}, \ldots, t_{n}\right\}} \prod_{i=2}^{n} t_{i}^{-1} d t_{2} \cdots d t_{n}=n !
$$


it follows that

$$
\frac{W_{1}}{\left|\lambda_{1}\right|} \prod_{i=1}^{n}\left|\lambda_{i}\right|^{\frac{1}{p_{i}}}=n ! \frac{1}{\left|\lambda_{1}\right|} \prod_{i=2}^{n}\left|\lambda_{i}\right|^{-1} \prod_{i=1}^{n}\left|\lambda_{i}\right|^{\frac{1}{p_{i}}}=n ! \prod_{i=1}^{n}\left|\lambda_{i}\right|^{\frac{1}{p_{i}}-1} .
$$

According to Theorem 1, we know that Theorem 2 holds.

Theorem 3 Suppose that $n \geq 2, \sum_{i=1}^{n} \frac{1}{p_{i}}=1\left(p_{i}>1\right), a>0, \lambda_{i}>0, \alpha_{i} \in \mathbf{R}, p_{i}>1+\alpha_{i}$. Then (i) the inequality

$$
\int_{\mathbf{R}_{+}^{n}} \frac{1}{\left(x_{1}^{\lambda_{1}}+x_{2}^{\lambda_{2}}+\cdots+x_{n}^{\lambda_{n}}\right)^{a}} \prod_{i=1}^{n} x_{i}^{-\frac{\alpha_{i}+1}{p_{i}}} d x_{1} \cdots d x_{n} \leq M \prod_{i=1}^{n}\left\|f_{i}\right\|_{p_{i}, \alpha_{i}}
$$

holds for some constant $M>0$ if and only if $\sum_{i=1}^{n} \frac{\alpha_{i}+1}{\lambda_{i} p_{i}}=-a+\sum_{i=1}^{n} \frac{1}{\lambda_{i}}$.

(ii) if (12) holds, then its best constant factor is

$$
\inf M=\frac{1}{\Gamma(a)} \prod_{i=1}^{n} \lambda_{i}^{\frac{1}{p_{i}}-1} \prod_{i=1}^{n} \Gamma\left(\frac{1}{\lambda_{i}}\left(1-\frac{\alpha_{i}+1}{p_{i}}\right)\right) .
$$

Proof Set $K\left(x_{1}, \ldots, x_{n}\right)=1 /\left(x_{1}^{\lambda_{1}}+x_{2}^{\lambda_{2}}+\cdots+x_{n}^{\lambda_{n}}\right)^{a}$, then $K\left(x_{1}, \ldots, x_{n}\right)$ is a quasi-homogeneous positive funct+ion with parameters $\left(-a, \lambda_{1}, \ldots, \lambda_{n}\right)$. By Lemma 2 ,

$$
\begin{aligned}
& W_{1}=\int_{\mathbf{R}_{+}^{n-1}} \frac{1}{\left(1+x_{2}^{\lambda_{2}}+\cdots+x_{n}^{\lambda_{n}}\right)^{a}} \prod_{i=2}^{n} x_{i}^{-\frac{\alpha_{i}+1}{p_{i}}} d x_{2} \cdots d x_{n} \\
& =\lim _{r \rightarrow+\infty} \int_{x_{i}>0, x_{2}^{\lambda_{2}}+\cdots+x_{n}^{\lambda_{n}} \leq r} \frac{1}{\left\{1+r\left[\left(\frac{x_{1}}{r^{1 / \lambda_{1}}}\right)^{\lambda_{1}}+\cdots+\left(\frac{x_{n}}{r^{1 / \lambda_{n}}}\right)^{\lambda_{n}}\right]\right\}^{n}} \\
& \times \prod_{i=2}^{n} x_{i}^{\left(1-\frac{\alpha_{i}+1}{p_{i}}\right)-1} d x_{2} \cdots d x_{n} \\
& =\lim _{r \rightarrow+\infty} \frac{r^{\sum_{i=2}^{n} \frac{1}{\lambda_{i}}-\sum_{i=2}^{n} \frac{1}{\lambda_{i}}\left(1-\frac{\alpha_{i}+1}{p_{i}}\right)} \prod_{i=2}^{n} \Gamma\left(\frac{1}{\lambda_{i}}\left(1-\frac{\alpha_{i}+1}{p_{i}}\right)\right)}{\prod_{i=2}^{n} \lambda_{i} \Gamma\left(\sum_{i=2}^{n} \frac{1}{\lambda_{i}}\left(1-\frac{\alpha_{i}+1}{p_{i}}\right)\right)} \\
& \times \int_{0}^{1} \frac{1}{(1+r t)^{a}} t^{\sum_{i=2}^{n} \frac{1}{\lambda_{i}}\left(1-\frac{\alpha_{i}+1}{p_{i}}\right)-1} d t \\
& =\lim _{r \rightarrow+\infty} \frac{\prod_{i=2}^{n} \Gamma\left(\frac{1}{\lambda_{i}}\left(1-\frac{\alpha_{i}+1}{p_{i}}\right)\right)}{\prod_{i=2}^{n} \lambda_{i} \Gamma\left(\sum_{i=2}^{n} \frac{1}{\lambda_{i}}\left(1-\frac{\alpha_{i}+1}{p_{i}}\right)\right)} \int_{0}^{r} \frac{1}{(1+u)^{a}} u^{\sum_{i=2}^{n} \frac{1}{\lambda_{i}}\left(1-\frac{\alpha_{i}+1}{p_{i}}\right)-1} d u \\
& =\frac{\prod_{i=2}^{n} \Gamma\left(\frac{1}{\lambda_{i}}\left(1-\frac{\alpha_{i}+1}{p_{i}}\right)\right)}{\prod_{i=2}^{n} \lambda_{i} \Gamma\left(\sum_{i=2}^{n} \frac{1}{\lambda_{i}}\left(1-\frac{\alpha_{i}+1}{p_{i}}\right)\right)} \int_{0}^{+\infty} \frac{1}{(1+u)^{a}} u^{\sum_{i=2}^{n} \frac{1}{\lambda_{i}}\left(1-\frac{\alpha_{i}+1}{p_{i}}\right)-1} d u \\
& =\frac{\prod_{i=2}^{n} \Gamma\left(\frac{1}{\lambda_{i}}\left(1-\frac{\alpha_{i}+1}{p_{i}}\right)\right)}{\prod_{i=2}^{n} \lambda_{i} \Gamma\left(\sum_{i=2}^{n} \frac{1}{\lambda_{i}}\left(1-\frac{\alpha_{i}+1}{p_{i}}\right)\right)} \\
& \times \frac{\Gamma\left(\sum_{i=2}^{n} \frac{1}{\lambda_{i}}\left(1-\frac{\alpha_{i}+1}{p_{i}}\right)\right) \Gamma\left(a-\sum_{i=2}^{n} \frac{1}{\lambda_{i}}\left(1-\frac{\alpha_{i}+1}{p_{i}}\right)\right)}{\Gamma(a)} \\
& =\frac{1}{\Gamma(a)} \prod_{i=2}^{n} \frac{1}{\lambda_{i}} \prod_{i=2}^{n} \Gamma\left(\frac{1}{\lambda_{i}}\left(1-\frac{\alpha_{i}+1}{p_{i}}\right)\right) \Gamma\left(\frac{1}{\lambda_{1}}\left(1-\frac{\alpha_{1}+1}{p_{1}}\right)\right)
\end{aligned}
$$




$$
=\frac{1}{\Gamma(a)} \prod_{i=2}^{n} \frac{1}{\lambda_{i}} \prod_{i=1}^{n} \Gamma\left(\frac{1}{\lambda_{i}}\left(1-\frac{\alpha_{i}+1}{p_{i}}\right)\right) .
$$

Based on this, we can obtain

$$
\frac{W_{1}}{\lambda_{1}} \prod_{i=1}^{n} \lambda_{i}^{\frac{1}{p_{i}}}=\frac{1}{\Gamma(a)} \prod_{i=1}^{n} \lambda_{i}^{\frac{1}{p_{i}}-1} \prod_{i=1}^{n} \Gamma\left(\frac{1}{\lambda_{i}}\left(1-\frac{\alpha_{i}+1}{p_{i}}\right)\right) .
$$

According to Theorem 1, we know that Theorem 3 holds.

\section{Funding}

This work is supported by the Guangdong Province Natural Science Foundation (No. 2015A030313896), the Characteristic Innovation Project (Natural Science) of Guangdong Province (Nos. 2015KTSCX097, 2016KTSCX094), the Science and Technology Program Project of Guangzhou (No. 201707010230).

\section{Competing interests}

The authors declare that they have no competing interests.

\section{Authors' contributions}

JC carried out the mathematical studies, participated in the sequence alignment, and drafted the manuscript. $\mathrm{BH}, \mathrm{YH}$, and BY participated in the design of the study and performed the numerical analysis. All authors read and approved the final manuscript.

\section{Authors' information}

Yong Hong (1959-), Professor, the major field of interest is in the area of inequality and harmonic analysis. Junfei Cao, email: jfcaomath@163.com. Bing He, email: hzs314@163.com. Bicheng Yang, email: bcyang@gdei.edu.cn.

\section{Author details}

${ }^{1}$ Department of Mathematics, Guangdong University of Education, Guangzhou, P.R. China. ${ }^{2}$ College of Mathematics and Statistics, Guangdong University of Finance and Economics, Guangzhou, P.R. China.

\section{Publisher's Note}

Springer Nature remains neutral with regard to jurisdictional claims in published maps and institutional affiliations.

Received: 1 April 2018 Accepted: 31 July 2018 Published online: 09 August 2018

\section{References}

1. He, B., Cao, J.F., Yang, B.C.: A brand new multiple Hilbert-type integral inequality. Acta Math. Sin., Chin. Ser. 58(4), 661-672 (2015)

2. Hong, Y.: A Hilbert-type integral inequality with quasi-homogeneous kernel and several functions. Acta Mathematics Sinica, Chinese Series 57(5), 833-840 (2014)

3. Perić, I., Vukvić, P.: Hardy-Hilbert's inequality with general homogeneous kernel. Math. Inequal. Appl. 12, 525-536 (2009)

4. Rassias, M., Yang, B.C.: On a Hardy-Hilbert-type inequality with a general homogeneous kernel. Int. J. Nonlinear Anal. Appl. 7(1), 249-269 (2016)

5. Chen, Q., Shi, Y., Yang, B.C.: A relation between two simple Hardy-Mulholland-type inequalities with parameters. J. Inequal. Appl. 2016, 75 (2016)

6. Hong, Y., Wen, Y.M.: A necessary and sufficient condition of that Hilbert type series inequality with homogeneous kernel and the best constant factor. Chin. Ann. Math. 37A(3), 329-336 (2016)

7. Yang, B.C., Chen, Q.: On a more accurate Hardy-Mulholland-type inequality. J. Inequal. Appl. 2016, 82 (2016)

8. Gao, M., Yang, B.C.: On the extended Hilbert's inequality. Proc. Am. Math. Soc. 126(3), $751-759$ (1998)

9. Yang, B.C.: On a more accurate multidimensional Hilbert-type inequality with parameters. Math. Inequal. Appl. 18, 429-441 (2015)

10. Xin, D.M., Yang, B.C., Chen, Q.: A discrete Hilbert-type inequality in the whole plane. J. Inequal. Appl. 2016, 133 (2016)

11. Kuang, J.C.: Applied Inequalities. Shangdong Science and Technology Press, Jinan (2010) (in Chinese)

12. Yang, B.C., Chen, Q.: On a Hardy-Hilbert-type inequality with parameters. J. Inequal. Appl. 2015, 339 (2015)

13. Yang, B.C., Chen, Q.: A new extension of Hardy-Hilbert's inequality in the whole plane. J. Funct. Spaces 2016, Article ID $9197476(2016)$

14. Hong, Y.: On multiple Hardy-Hilbert integral inequalities with some parameters. J. Inequal. Appl. 2006, Article ID $94960(2006)$

15. Huang, Q.L., Yang, B.C.: On a multiple Hilbert-type integral operator and applications. J. Inequal. Appl. 2009, Article ID 192197 (2009)

16. Hardy, G.H., Littlewood, J.E., Pólya, G.: Inequalities. Cambridge University Press, Gambridge (1952)

17. Yang, B.C., Debnath, L.: Half-Discrete Hilbert-Type Inequalities. World Scientific, Singapore (2014)

18. Kuang, J., Debnath, L.: On new generalizations of Hilbert's inequality and their applications. J. Math. Anal. Appl. 245(1), 248-265 (2000) 
19. Rassias, M., Yang, B.C.: On a Hilbert-type integral inequality related to the extended Hurwitz zeta function in the whole plane. Acta Appl. Math. 2018, 1-14 (2018)

20. Rassias, M., Yang, B.C.: Equivalent properties of a Hilbert-type integral inequality with the best constant factor related to the Hurwitz zeta function. Ann. Funct. Anal. 9(2), 282-295 (2018)

21. Rassias, M., Yang, B.C.: A half-discrete Hilbert-type inequality in the whole plane related to the Riemann zeta function. Appl. Anal. 2017(2), 1-21 (2017)

22. Rassias, M., Yang, B.C.: A Hilbert-type integral inequality in the whole plane related to the hypergeometric function and the beta function. J. Math. Anal. Appl. 428(2), 1286-1308 (2015)

23. Rassias, M., Yang, B.C.: On a multidimensional Hilbert-type integral inequality associated to the gamma function. Appl. Math. Comput. 249, 408-418 (2014)

24. Milovanović, G.V., Rassias, M.: Analytic Number Theory, Approximation Theory and Special Functions. Springer, New York (2014)

25. Fichtingoloz, G.M.: A Course in Differential and Integral Calculus. Renmin Jiaoyu Publishers, Beijing (1957) (in Chinese)

Submit your manuscript to a SpringerOpen ${ }^{\circ}$ journal and benefit from:

- Convenient online submission

- Rigorous peer review

- Open access: articles freely available online

- High visibility within the field

- Retaining the copyright to your article

Submit your next manuscript at $\gg$ springeropen.com 